Pacific Journal of Mathematics

POWER SERIES RINGS OVER PRÜFER DOMAINS 


\title{
POWER SERIES RINGS OVER PRÜFER DOMAINS
}

\author{
JIMMY T. ARNOLD
}

Let $R$ be a commutative ring with identity. $R$ is said to have dimension $n$, written $\operatorname{dim} R=n$, if there exists a chain $P_{0} \subset P_{1} \subset \cdots \subset P_{n}$ of $n+1$ prime ideals of $R$, where $P_{n} \subset R$, but no such chain of $n+2$ prime ideals. Seidenberg has shown that if $\operatorname{dim} R=n$ and $X$ is an indeterminate over $R$, then $n+1 \leqq \operatorname{dim} R[X] \leqq 2 n+1$. Moreover, he has shown that $\operatorname{dim} R[X]=n+1$ if $R$ is a Prüfer domain. The author has shown that if $V$ is a rank one nondiscrete valuation ring, then $\operatorname{dim} V[[X]]=\infty$. The principal result of this paper is that if $D$ is a Prïfer domain with $\operatorname{dim} D=n$, then either $\operatorname{dim} D[[X]]=n+1$ or $\operatorname{dim} D[[X]]=\infty$, and necessary and sufficient conditions are given.

1. Notation. Our notation and terminology are essentially that of [4]. Throughout, $R$ denotes a commutative ring with identity and $T$ denotes the total quotient ring of $R$. By an overring $S$ of $R$, we shall mean a ring $S$ such that $R \subseteq S \subseteq T$. The set of natural numbers will be denoted by $\omega$ and $\omega_{0}$ is the set of nonnegative integers. If $A$ is an ideal of $R$, then we let

$$
A[[X]]=\left\{f(X)=\sum_{i=0}^{\infty} a_{i} X^{i} / a_{i} \in A \text { for each } i \in \omega_{0}\right\}
$$

and we define $A R[[X]]$ to be the ideal of $R[[X]]$ which is generated by $A$. The ideal $A$ will be called an SFT-ideal (an ideal of strong finite type) provided there exists a finitely generated ideal $B \subseteq A$ and $k \in \omega$ such that $a^{k} \in B$ for each $a \in A$. We say that $R$ is an SFT-ring provided each ideal of $R$ is an SFT-ideal.

2. Some properties of SFT-rings. Arnold has shown in [1] that if $R$ is not an SFT-ring, then $\operatorname{dim} R[[X]]=\infty$. In this paper we are primarily concerned with finite-dimensional Prüfer domains which are also SFT-rings, and our main result shows that for such a domain $D$, if $\operatorname{dim} D=n$, then $\operatorname{dim} D[[X]]=n+1$. Before restricting our attention to Prüfer domains, however, we wish to consider some properties of arbitrary SFT-rings.

LEMma 2.1. If $A_{1}, A_{2}$ are SFT-ideals of $R$ and if $C$ is an ideal of $R$ such that $A_{1} \cap A_{2} \supseteqq C \supseteqq A_{1} A_{2}$, then $C$ is an SFT-ideal.

Proof. For $i=1,2$, there exists a finitely generated ideal 
$B_{i} \subseteq A_{i}$ and $k_{i} \in \omega$ such that $a_{i}^{k_{i}} \in B_{i}$ for each $a_{i} \in A_{i}$. Set $k=k_{1}+k_{2}$. Then for $c \in C$, we have that $c^{k}=c^{k_{1}} c^{k_{2}} \in B_{1} B_{2} \subseteq A_{1} A_{2} \subseteq C$. Since $B_{1} B_{2}$ is finitely generated, the lemma follows.

Proposition 2.2. $R$ is an SFT-ring if and only if each prime ideal of $R$ is an SFT-ideal.

Proof. Suppose that $R$ is not an SFT-ring. It follows from a straight-forward application of Zorn's Lemma that $R$ contains an ideal $P$ which is maximal among those ideals of $R$ which are not SFT-ideals. Thus, if $A$ and $B$ are ideals of $R$ which properly contain $P$, then $A$ and $B$ are SFT-ideals. It is an immediate consequence of Lemma 2.1 that $P \nsupseteq A B$, for otherwise, $P$ would be an SFT-ideal. Therefore, $P$ is a prime ideal of $R$ which is not an SFT-ideal.

Proposition 2.3. If the ring $S$ is the homomorphic image of an $S F T$-ring $R$, then $S$ is also an SFT-ring.

The proof of Proposition 2.3 is straightforward and will be omitted.

Before stating our next result, we recall that an overring $R_{1}$ of $R$ is called a flat overring of $R$ provided $R_{1}$ is flat as an $R$-module. Richman in [8] has studied flat overrings of integral domains and has dubbed them "generalized quotient rings" due to the fact that many of the classical properties of quotient rings also hold for flat overrings. Flat overrings are further considered in [2], where they are shown to be a special class of "generalized transforms." Specifically, if $R_{1}$ is a flat overring of $R$, then there exists a multiplicatively closed set $\mathscr{S}$ of ideals of $R$ such that

$$
R_{1}=R_{\mathscr{S}}=\{\xi \in T / \xi A \subseteq R \text { for some } A \in \mathscr{S}\} \text {. }
$$

Moreover, $\mathscr{S}$ may be chosen so that $A R_{1}=R_{1}$ for each $A \in \mathscr{S}[2$, Thm. 1.3]. Using this notation and terminology, we now prove the following result.

Proposition 2.4. Let $R$ be an SFT-ring. If $R_{1}$ is a flat overring of $R$, then $R_{1}$ is an SFT-ring.

Proof. Let $R_{1}=R_{\mathscr{S}}$ as described above, and let $Q$ be a prime ideal of $R$. If we set $P=Q \cap R$, then $Q=P_{S}$ [2, Thm. 1.1]; thus, for $q \in Q$, there exists $A \in \mathscr{S}$ such that $q A \subseteq P$. But $P$ is an SFTideal, so there is a finitely generated ideal $B \subseteq P$ and $k \in \omega$ such 
that $p^{k} \in B$ for each $p \in P$. In particular, $q^{k} a^{k} \in B$ for each $a \in A$. Let $\mathscr{A}=\left\{\xi \in R_{1} / q^{k} \xi \in B R_{1}\right\}$. Then $\mathscr{A}$ is an ideal of $R_{1}$ and $a^{k} \in \mathscr{A}$ for each $a \in A$. Consequently, we have that $\sqrt{\mathscr{A}}=\sqrt{A R_{1}}=R_{1}$, from which it is immediate that $\mathscr{A}=R_{1}$. This shows that $q^{k} \in B R_{1}$ for each $q \in Q$, and hence, that $Q$ is an SFT-ideal in $R_{1}$.

Proposition 2.5. If $R$ is an SFT-ring, then $R$ satisfies the ascending chain condition for radical ideals, i.e., the prime spectrum of $R$ is Noetherian.

Proof. Clearly, each radical ideal of $R$ is the radical of a finitely generated ideal. But this is equivalent to the ascending chain condition for radical ideals [7, p. 633].

If $R$ satisfies the ascending chain condition for radical ideals, then it is shown in [6, p. 59] that each ideal of $R$ has only finitely many minimal prime divisors. As an immediate consequence we have

COROLLARY 2.6. Each ideal of an SFT-ring has only finitely many minimal prime divisors.

We conclude this section with the following lemma.

LEMma 2.7. Let $D$ be an integral domain which is an SFT-ring. If $P$ is a nonzero prime ideal of $D$, then $P \neq P^{2}$.

Proof. Let $V$ be a valuation overring of $D$ for which $P V \neq V$. Since $P$ is an SFT-ideal, there exists a finitely generated ideal $B \subseteq P$ and $k \in \omega$ such that $p^{k} \in B$ for each $p \in P$. If $P_{1}=P V$ and $B_{1}=B V$, then we also have $\xi^{k} \in B_{1}$ for each $\xi \in P_{1}$. Since $V$ is a valuation ring, it follows that $P_{1}^{k} \leqq B_{1} \leqq P_{1}$. If $B_{1}=P_{1}$, then $P_{1}$ is principal, so $P_{1} \neq P_{1}^{2}$. If $B_{1} \subset P_{1}$, then $P_{1}^{k} \neq P_{1}$, and again it follows that $P_{1} \neq P_{1}^{2}$. Consequently, $P \neq P^{2}$ as we wished to show.

3. Prüfer domains which are SFT-rings. Throughout this section $D$ will denote a Prüfer domain. We begin by giving a characterization of those Prüfer domains which are also SFT-rings.

Propositon 3.1. In order that the Prïfer domain $D$ be an SFTring, it is necessary and sufficient that for each nonzero prime ideal $P$ of $D$, there exists a finitely generated ideal $A$ such that

$$
P^{2} \subseteq A \subseteq P
$$


Proof. In view of Proposition 2.2, it is clear that the given conditions are sufficient to insure that an arbitrary ring is an SFTring. To show that they are also necessary for the Prüfer domain $D$, suppose that $D$ is an SFT-ring and let $P$ be a nonzero prime ideal of $D$. Since $P$ is an SFT-ideal, $P=\sqrt{B}$ for some finitely generated ideal $B$ of $D$. By Lemma 2.7 there exists $p \in P-P^{2}$. If we set $A=B+(p)$, then $A$ is finitely generated, $P=\sqrt{A}$, and $P^{2} \nsupseteq A$. Let $M$ be a maximal ideal of $D$ which contains $P$. Since $P^{2}$ is $P$ - primary $[4,19.3]$, we have $P^{2}=P^{2} D_{M} \cap D$. It follows that $P^{2} D_{M} \supseteqq A D_{M}$; hence $P^{2} D_{M} \subseteq A D_{M}$. Consequently, $P^{2} \subseteq A \leqq P$.

CoRollary 3.2. Suppose that $D$ is an SFT-ring, let $P$ be a nonzero prime ideal of $D$ and let $p \in P-P^{2}$. For each $n \in \omega$ there exists $s_{n} \in D-P$ such that $s_{n} P^{n+1} \subseteq\left(p^{n}\right)$.

Proof. Let $A=\left(a_{1}, \cdots, a_{m}\right)$ be a finitely generated ideal of $D$ such that $P^{2} \subseteq A \subseteq P$. Then $A D_{P} \subseteq P D_{P}=(p) D_{P}$, so we may find $s \in D-P$ such that $s a_{i} \in(p)$ for $1 \leqq i \leqq m$. For each $n \in \omega$, set $s_{n}=s^{n}$. For $n=1$ we get $s_{1} P^{2} \subseteq s_{1} A \leqq(p)$, and for $n>1$ we get $s_{n} P^{n+1}=\left(s_{1} P^{2}\right)\left(s_{n-1} P^{n-1}\right) \subseteq(p)\left(s_{n-1} P^{n-1}\right) \subseteq s_{n-1} P^{n}$. The corollary follows by induction on $n$.

Hereafter, we assume that $D$ has finite dimension; $\Pi=\left\{P_{\alpha}\right\}_{\alpha \in A}$ is the set of minimal prime ideals for $D$, and $\mathscr{C}=\left\{M_{\beta}\right\}_{\beta \in \Gamma}$ is the set of maximal ideals of $D$.

If $D$ is an SFT-ring, then as an immediate consequence of Lemma 2.7, we see that $D_{Q}$ is a discrete valuation ring for each prime ideal $Q$ of $D[4, \mathrm{p} .177]$. In particular, $D_{P_{\alpha}}$ is a rank one discrete valuation ring for each $P_{\alpha} \in \Pi$. Dedekind domains and discrete valuation rings with finite dimension provide immediate examples of Prüfer domains wich are SFT-rings. In fact, if $\operatorname{dim} D=1$, then it follows from $[4,30.2]$ that $D$ is an SFT-ring if and only if $D$ is a Dedekind domain. If we set $D^{\prime}=\bigcap_{\alpha} D_{P_{\alpha}}$, then from [4, 22.1], we see that $D^{\prime}$ is a Prüfer domain. Richman shows in [8] that each overring of a Prüfer domain is a flat overring, so by Proposition $2.4 D^{\prime}$ is an SFTring. It is immediate from Corollary 2.6 that $D^{\prime}$ has finite real character [cf. 4, p. 505], so by $\left[4,35.8\right.$ ] we have $\operatorname{dim} D^{\prime}=1$. Our preceding remarks now imply that $D^{\prime}$ is a Dedekind domain. By $[4,36.11], D^{\prime}[[X]]$ is a Krull domain, and since $D^{\prime}$ is Noetherian we have that $\operatorname{dim} D^{\prime}[[X]]=2[3, \mathrm{p} .603]$. But the maximal ideals of $D^{\prime}[[X]]$ are of the form $P+(X)$, where $P$ is a maximal ideal of $D^{\prime}$, so $J^{\prime}=$ $\left(D^{\prime}[[X]]\right)_{D^{\prime}-(0)}$ is a one-dimensional Krull domain - that is, $J^{\prime}$ is a Dedekind domain $[4,35.16]$. Set $J=(D[[X]])_{D-(0)}$ and let $L$ denote quotient field of $D[[X]]$. 
Lemma 3.3. If $D$ is an SFT-ring, then $J=J^{\prime} \cap L$.

Proof. Clearly, $J \subseteq J^{\prime} \cap L$, so let $\xi(X)=f(X) / g(X) \in J^{\prime} \cap L$, where $f(X), g(X) \in D[[X]], f(X)=\sum_{i=0}^{\infty} f_{i} X^{i}$ and $g(X)=\sum_{i=0}^{\infty} g_{i} X^{i}$. Since $\xi(X) \in J^{\prime}$, there exist $\lambda \in D^{\prime}-(0)$ and $h(X) \in D^{\prime}[[X]]$ such that $\lambda f(X)=g(X) h(X)$. Consequently, if there exists $m \in \omega_{0}$ such that $g_{i}=0$ for $i \leqq m$, then we also have that $f_{i}=0$ for $i \leqq m$. Therefore, in our representation $\xi(X)=f(X) / g(X)$, we may assume that $g_{0} \neq 0$. If $M_{\beta} \in \mathscr{C}$ is such that $g_{0} \notin M_{\beta}$, then $g(X)$ is a unit in $D_{M_{\beta}}[[X]]$. Thus, $\xi(X) \in D_{M_{\beta}}[[X]]$. Let $Q$ be a minimal prime divisor of $\left(g_{0}\right) D$ and let $P$ be the minimal prime ideal of $D$ contained in $Q$. Clearly, $\xi(X) \in\left(D_{P}[[X]]\right)_{D_{P^{-}}(0)}$ and by [3, p. 602], $\left(D_{P}[[X]]\right)_{D_{P^{-}}(0)}=\left(D_{Q}[[X]]\right)_{D_{Q^{-}}(0)}$. Hence, there exists $d \in D_{Q}-(0)$ such that $d \xi(X) \in D_{Q}[[X]]$. In fact, we may assume that $d \in D$. If $M_{\beta} \in \mathscr{K}$ is such that $M_{\beta} \supseteqq Q$, then $Q D_{Q} \subseteq D_{M_{3}}[4,14.6]$. Consequently, for $q \in Q-(0)$, we have

$$
q d \xi(X) \in D_{M_{\beta}}[[X]] \text {. }
$$

By Corollary 2.6, $\left(g_{0}\right) D$ only finitely many minimal prime divisors, so it follows that we may find $r \in D-(0)$ such that

$$
r \xi(X) \in \bigcap_{\beta} D_{M_{\beta}}[[X]]=D[[X]] .
$$

Therefore, $\xi(X) \in J$ as we wished to show.

We wish to show that $J$ is, in fact, a Dedekind domain. In order to do this, we first need to consider the domain

$$
U=D^{\prime}[[X]] \cap L .
$$

LEMmA 3.4. If $A$ is an ideal of $U$ such that $A D^{\prime}[[X]]$ is contained in no minimal prime ideal of $D^{\prime}[[X]]$, then $X^{n} \in A$ for some $n \in \omega$.

Proof. The only possible minimal prime divisors for $A D^{\prime}[[X]]$ are of the form $P+(X)$, where $P$ is a maximal ideal of $D^{\prime}$. Consequently, $X \in \sqrt{A D^{\prime}[[X]]}$ - that is there exists $n \in \omega$ such that $X^{n} \in A D^{\prime}[[X]]$. Let $\lambda_{1}(X), \cdots, \lambda_{k}(X) \in D^{\prime}[[X]]$ and $a_{1}(X), \cdots, a_{k}(X) \in A$ be such that $X^{n}=\sum_{i=1}^{k} \lambda_{i}(X) a_{i}(X)$. If $\lambda_{i}(X)=\sum_{j=0}^{\infty} \beta_{i j} X^{j}$, set $\gamma_{i}(X)=$ $\sum_{j=0}^{n} \beta_{i j} X^{j}$ and $\zeta_{i}(X)=\left(\lambda_{i}(X)-\gamma_{i}(X)\right) / X^{n+1}$. For $1 \leqq i \leqq k$ we have that $\gamma_{i}(X) \in D^{\prime}[[X]] \cap L=U$, and hence,

$$
\begin{aligned}
\sum_{i=1}^{l} \gamma_{i}(X) a_{i}(X) & =\sum_{i=1}^{k} \lambda_{i}(X) a_{i}(X)-X^{n+1}\left(\sum_{i=1}^{k} \zeta_{i}(X) a_{i}(X)\right) \\
& =X^{n}-X^{n+1} \sum_{i=1}^{k} \zeta_{i}(X) a_{i}(X) \in A
\end{aligned}
$$


But $u(X)=1-X\left(\sum_{i=1}^{k} \zeta_{i}(X) a_{i}(X)\right) \in U$ is a unit in $D^{\prime}[[X]]$, so it is also a unit in $U$. Therefore, $X^{n} \in A$.

Assuming that $D$ is an SFT-ring, we know that $D_{P_{\alpha}}$ is a rank one discrete valuation ring for each $P_{\alpha} \in \Pi$. If $v$ is a valuation associated with $D_{P_{\alpha}}$, then we may define a "trivial extension" $v^{*}$ of $v$ to $L$ by setting

$$
v^{*}\left(\sum_{i=0}^{\infty} h_{i} x^{i}\right)=\min _{i \in \omega_{0}}\left\{v^{*}\left(h_{i}\right) \mid h_{i} \neq 0\right\}
$$

for $\sum_{i=0}^{\infty} h_{i} X^{i} \in D[[X]][5, \mathrm{p} .380]$. If $V^{*}$ is the valuation overring of $D[[X]]$ associated with $v^{*}$, then $V^{*}$ is rank one discrete and is centered on $P_{\alpha}[[X]]$ in $D[[X]]$. Since $D^{\prime} \subseteq D_{p_{\alpha}}$, we may also extend $D_{P_{\alpha}}$ to a rank one discrete valuation overring $V_{1}^{*}$ of $D^{\prime}[[X]]$. If $P_{1}=P_{\alpha} D_{P_{\alpha}} \cap D^{\prime}$, then $V_{1}^{*}$ is the essential valuation overring of $D^{\prime}[[X]]$ associated with the minimal prime ideal $P_{1}[[X]][5, \mathrm{p} .380]$. It follows from $[4,36.10]$ that $U=D^{\prime}[[X]] \cap L$ is a Krull domain and each minimal prime ideal of $U$ has the form $Q \cap U$, where $Q$ is a minimal prime ideal of $D^{\prime}[[X]]$. Moreover, whenever $Q$ is a minimal prime ideal of $D^{\prime}[[X]]$ such that $Q \cap U \neq(0)$, then $Q \cap U$ is a minimal prime ideal of $U$ and $\left(D^{\prime}[[X]]\right)_{Q} \cap L$ is the essential valuation overring of $U$ associated with $Q \cap U$. In particular, $V^{*}=$ $V_{1}^{*} \cap L$ is the essential valuation overring of $U$ associated with $P_{1}^{\prime}=P_{1}[[X]] \cap U$. We are now in a position to prove the following key result.

Proposition 3.5. Suppose that $D$ is an SFT-ring and let $P_{\alpha} \in \Pi$. Then $P_{\alpha}[[X]]$ is a minimal prime ideal of $D[[X]]$.

Proof. Let $Q$ be a nonzero prime ideal of $D[[X]], Q \subseteq P_{\alpha}[[X]]$. If $(0) \subset Q \cap D \subseteq P_{\alpha}=P_{\alpha}[[X]] \cap D$, then $Q \cap D=P_{\alpha}$. Consequently, $Q \supseteqq P_{\alpha} D[[X]]$. But $P_{\alpha}[[X]]=\sqrt{P_{\alpha} D[[X]]}[1, \mathrm{Thm} .1]$, so it follows that $Q=P_{\alpha}[[X]]$. Thus, we may assume that $Q \cap D=(0)$. Let $W$ be a valuation overring of $D[[X]]$ with prime ideals $Q_{1} \supset Q_{2}$ such that $Q_{1}$ is maximal in $W, Q_{1} \cap D[[X]]=P_{\alpha}[[X]]$ and $Q_{2} \cap D[[X]]=Q$. If $p \in P_{\alpha}-P_{\alpha}^{2}$, then $p \in Q_{1}-Q_{2}$, so we may assume that $Q_{1}=\sqrt{p W}$. We wish to show that $U \subseteq W$. Thus, let $\xi(X) \in U$. Since

$$
U \subseteq J^{\prime} \cap L=J,
$$

we may write $\xi(X)=f(X) / d$, where $f(X) \in D[[X]]$ and $d \in D-(0)$. Suppose that $\xi(X) \notin W$. Then

$$
\xi(X)^{-1}=d / f(X) \in Q_{1}, \quad \text { so } \quad w(d)>w(f(X)) \geqq 0,
$$

where $w$ is a valuation associated with $W$. Now $w(d)>0$ implies 
that $d \in P_{\alpha}$ and hence, that $v^{*}(d)>0$. Since $\xi(X) \in U \subseteq V^{*}$, we have that $v^{*}(\xi(X)) \geqq 0$ - that is, $v^{*}(f(X)) \geqq v^{*}(d)>0$. If $v^{*}(d)=k$, then $(d) D_{P_{\alpha}}=P_{\alpha}^{k} D_{P_{\alpha}}=\left(p^{k}\right) D_{P_{\alpha}}$, so there exists $s, t \in D-P_{\alpha}$ such that $s d=t p^{k}$. But then $v^{*}(f(X)) \geqq k$, so $f(X) \in P_{\alpha}^{k}[[X]]$. Since $Q_{1}=$ $\sqrt{p W}$, there exists $n \in \omega$ such that $d^{n} / f(X)^{n}=p \lambda(X)$ for some $\lambda(X) \in W$. But $f(X)^{n} \in P_{\alpha}^{n k t}[[X]]$, so by Corollary 3.2, there exists $\tau \in D-P_{\alpha}$ such that $\tau(f(X))^{n}=p^{n k-1} f_{1}(X)$, where $f_{1}(X) \in D[[X]]$. Since $n k \leqq v^{*}\left(\tau f(X)^{n}\right)=v^{*}\left(p^{n k-1} f_{1}(X)\right)=(n k-1)+v^{*}\left(f_{1}(X)\right)$, it follows that $f_{1}(X) \in P_{\alpha}[[X]]$. We now have

$$
\begin{aligned}
\lambda(X) & =d^{n} / p(f(X))^{n}=\tau s^{n} d^{n} / s^{n} p \tau(f(X))^{n} \\
& =\tau t^{n} p^{n k} / s^{n} p^{n k} f_{1}(X)=\tau t^{n} / s^{n} f_{1}(X) .
\end{aligned}
$$

But $w\left(\tau t^{n} / s^{n} f_{1}(X)\right)=-w\left(f_{1}(X)\right)<0$, so it must be the case that $W \supseteqq U$.

Let $P_{1}^{\prime}$ be the center of $V^{*}$ on $U$ - that is, $P_{1}^{\prime}=P_{1}[[X]] \cap U$, and let $Q_{1}^{\prime}$ be the center of $W$ on $U$. We claim that $Q_{1}^{\prime} \supseteqq P_{1}^{\prime}$, for let $\xi(X)=f(X) / d \in P_{1}^{\prime}$. Then $v^{*}(f(X))>v^{*}(d) \geqq 0$; in particular,

$$
f(X) \in P_{\alpha}[[X]] \subseteq Q_{1} .
$$

If $d \notin P_{\alpha}$, then $w(\xi(X))=w(f(X))>0$ and hence, $\xi(X) \in Q_{1} \cap U=Q_{1}^{\prime}$. Thus, assume that $d \in P_{\alpha}$ - say $v^{*}(d)=k$. Then arguing as above, there exist $s, t \in D-P_{\alpha}$ such that $s d=t p^{k}$. Moreover,

$$
v^{*}(f(X)) \geqq k+1, \quad \text { so } f(X) \in P_{\alpha}^{k+1}[[X]] \text {. }
$$

Consequently, there exists $\tau \in D-P_{\alpha}$ and $f_{1}(X) \in P_{\alpha}[[X]]$ such that $\tau f(X)=p^{k} f_{1}(X)$. This yields $\xi(X)=s \tau f(X) / \tau s d=s p^{k} f_{1}(X) / \tau t p^{k}=$ $s f_{1}(X) / \tau t$ which, as we have just observed, is in $Q_{1}^{\prime}$. Therefore, $P_{1}^{\prime} \subseteq Q_{1}^{\prime}$. But we also have that $Q_{2} \cap U \subset Q_{1}^{\prime}$, and $Q_{2} \cap U \nsupseteq P_{1}^{\prime}$ since $\left(Q_{2} \cap U\right) \cap D[[X]]=Q \subset P_{\alpha}[[X]]=P_{1}^{\prime} \cap D[[X]]$. It follows that $Q_{1}^{\prime}$ contains at least two distinct minimal prime ideals of $U$ and hence, $\left(Q_{1}^{\prime}\right) D^{\prime}[[X]]$ cannot be contained in any minimal prime ideal of $D^{\prime}[[X]]$. By Lemma 3.4, there exists $n \in \omega$ such that $X^{n} \in Q_{1}^{\prime} \subseteq Q_{1}$, contrary to our assumption that $Q_{1} \cap D[[X]]=P_{\alpha}[[X]]$. We conclude that $P_{\alpha}[[X]]$ is minimal in $D[[X]]$.

We now digress momentarily in order to strengthen the results of Proposition 3.5. It follows from $[4,16.10]$ that if $P$ is a prime ideal of $D$, then each prime ideal of $D[X]$ contained in $P[X]$ is the extension of a prime ideal of $D$. We show that the following analogue holds in $D[[X]]$.

CoROLLARY 3.6. If $D$ is an SFT-ring and if $P$ is a prime ideal of $D$, then the only prime ideals of $D[[X]]$ contained in $P[[X]]$ have 
the form $P_{1}\left[[X]\right.$ for some prime ideal $P_{1}$ of $D$.

Proof. Suppose that $D$ is an SFT-ring, let $P$ be a prime ideal of $D$, and let $Q$ be a nonzero prime ideal of $D[[X]]$ such that $Q \subset P[[X]]$. If $Q \cap D=P^{\prime} \neq(0)$, then $Q \supseteq \sqrt{P^{\prime} D[[X]]}=P^{\prime}[[X]]$ $\left[1\right.$, Thm. 1], so by considering $D[[X]] / P^{\prime}[[X]] \cong\left(D / P^{\prime}\right)[[X]]$, we may assume that $Q \cap D=(0)$. In view of Proposition 3.5, we may also assume that $P$ is not minimal in $D$; hence, there exists a prime ideal $P_{1}$ of $D$ such that $P_{1} \subset P$ and there are no prime ideals properly contained between $P$ and $P_{1}$ [4,19.3]. We further assume that $P_{1}[[X]] \nsupseteq Q$. Let $p \in P-P^{2}$ and $p_{1} \in P_{1}-P_{1}^{2}$.

$$
S=\left\{p^{k} s(X) \mid k \in \omega_{0}, s(X) \in D[[X]]-P[[X]]\right\}
$$

is a multiplicative system in $D[[X]]$ and $Q \cap S=\phi$. Let

$$
f(X) \in Q-P_{1}[[X]]
$$

and set $A=\left(f(X), p_{1}\right) D[[X]]$. Suppose that $r(X), t(X) \in D[[X]]$ are such that $r(X) f(X)+p_{1} t(X)=p^{k} s(X) \in S$. Since $P_{1} D_{P}=\bigcap_{n=1}^{\infty} P^{n} D_{P}$ $[4,14.1]$, there exist $y, z \in D, y \notin P, z \in P_{1}$, such that $y p_{1}=z p^{k}$. Therefore, $\operatorname{yr}(X) f(X)=p^{k}(y s(X)-z t(X)) \in S$, contrary to the fact that $Q \cap S=\phi$. Thus, $A \cap S=\phi$, and there exists a prime ideal $Q_{1}$ of $D[[X]]$ such that $A \subseteq Q_{1}$ and $Q_{1} \cap S=\phi$. Clearly, $Q_{1} \subset P[[X]]$, and since $p_{1} \in Q_{1} \cap D \subset P[[X]] \cap D$, it follows that $Q_{1} \cap D=P_{1}$. But then we have $P_{1}[[X]] \subset Q_{1} \subset P[[X]]$ which yields, on reducing to $\left(D / P_{1}\right)[[X]]$, a contradiction to Proposition 3.5. We conclude that no such $Q$ exists.

Proposition 3.7. If $D$ is an SFT-ring, then $J$ is a Dedekind domain.

Proof. Since $J=J^{\prime} \cap L, J$ is a Krull domain [4, 36.10]. Therefore, it suffices to show that $\operatorname{dim} J=1[4,35.16]$. Let $Q J$ be a nonzero prime ideal of $J$, where $Q$ is a prime ideal in $D[[X]]$ such that $Q \cap D=(0)$. We first suppose that $Q D^{\prime}[[X]]$ is contained in some minimal prime ideal $Q^{\prime}$ of $D^{\prime}[[X]]$. We cannot have $Q^{\prime}=P_{1}[[X]]$ for any prime ideal $P_{1}$ of $D^{\prime}$, for if we set $P_{\alpha}=P_{1} \cap D$, then

$$
P_{1}[[X]] \cap D\left[\left[X[]=P_{\alpha}[[X]]\right.\right. \text {. }
$$

But $P_{\alpha} \in \Pi$, so by Proposition 3.5, $P_{\alpha}[[X]]$ is minimal in $D[[X]]$. Since we must have $Q \subseteq Q D^{\prime}[[X]] \cap D[[X]] \subseteq Q^{\prime} \cap D[[X]]$, it follows that $Q^{\prime} \cap D^{\prime}=(0)$. Consequently, $Q^{\prime} J^{\prime}$ is a minimal prime ideal of $J^{\prime}$. Since $Q^{\prime} J^{\prime} \cap J \supseteqq Q J \neq(0), Q J$ is a minimal prime ideal in $J$. 
We now consider the possibility that $Q D^{\prime}[[X]]=(Q U) D^{\prime}[[X]]$ is contained in no minimal prime ideal of $D^{\prime}[[X]]$. But if this is the case, then by Lemma 3.4, $X^{n} \in Q U$ for some $n \in \omega$. Since

$$
Q=Q J \cap D[[X]]=(Q U) J \cap D[[X]],
$$

it follows that $X \in Q$. But $Q \neq(X) D[[X]]$, for $(X) D^{\prime}[[X]]$ is a minimal prime ideal of $D^{\prime}[[X]]$ and clearly, $(X) D^{\prime}[[X]] \supseteqq(X) D[[X]]$. Therefore, $Q \supset(X) D[[X]]$, from which it is immediate that $Q \cap D \neq(0)$. Since this contradicts our assumption on $Q$, we conclude that $Q J$ is minimal in $J$.

We now state the principal result of this paper.

Theorem 3.8. Let $D$ be Prïfer domain with $\operatorname{dim} D=n$. The following statements are equivalent:

(1) $D$ is an SFT-ring.

(2) $\operatorname{Dim} D[[X]]=n+1$.

(3) $\operatorname{Dim} D[[X]]<\infty$.

Proof. It is clear that (2) implies (3) and it is shown in [1] that (3) implies (1). We show that (1) implies (2) by induction of $n$. But if $n=1$, then $D$ is a Dedekind domain, so the theorem holds. Now suppose that $\operatorname{dim} D=n>1$ and let $(0) \subset Q_{1} \subset \cdots \subset Q_{k}, k>1$, be a chain of prime ideals of $D[[X]]$. Since $Q_{2}$ is not minimal, it follows from Proposition 3.7 that $Q_{2} \cap D \neq(0)$. In particular, $Q_{2} \cap D \supseteqq P_{\alpha}$ for some $P_{\alpha} \in \Pi$. But then $Q_{2} \supseteqq \sqrt{P_{\alpha} D[[X]]}=P_{\alpha}[[X]][1$, Thm. 1], and the containment is proper since $P_{\alpha}[[X]]$ is minimal in $D[[X]]$. This yields a chain $(0) \subset Q_{2} / P_{\alpha}[[X]] \subset \cdots \subset Q_{k} / P_{\alpha}[[X]]$ of $k-1$ prime ideals in $D[[X]] / P_{\alpha}[[X]] \cong\left(D / P_{\alpha}\right)[[X]]$. Since $D / P_{\alpha}$ is a Prüfer domain $[4,18.5]$ which is, by Proposition 2.3, an SFT-ring, our induction hypothesis implies that $k-1 \leqq n$. Consequently, $k \leqq n+1$. But we already know that $\operatorname{dim} D[[X]] \geqq n+1$, so equality must hold.

4. Example. Suppose that $\operatorname{dim} R=n$. We have seen that

$$
\operatorname{dim} R[X]=n+1 \nRightarrow \operatorname{dim} R[[X]]=n+1 ;
$$

for if $D$ is any $n$-dimensional Prüfer domain which is not an SFTring, then $\operatorname{dim} D[X]=n+1$ while $\operatorname{dim} D[[X]]=\infty$. We now give an example which shows that

$$
\operatorname{dim} R[[X]]=n+1 \Rightarrow \operatorname{dim} R[X]=n+1 .
$$

Thus, let $F$ be a field and $K=F(Y)$ a simple transcendental extension of $F$. Let $V=K+M$ be a rank one discrete valuation ring with maximal ideal $M$ (e.g., take $V=K[[Z]])$ and set $D=F+M$. 
Then $D$ is integrally closed and $M$ is the unique nonzero prime ideal of $D$ [4,App. 2]. In particular, $\operatorname{dim} D=1$. But $D$ is not a Prüfer domain, so $\operatorname{dim} D[X]=3[4,25.13]$. For $m \in M$, we have

$$
m V[[X]] \subseteq M[[X]] \subseteq D[[X]],
$$

whence it follows that $(V[[X]])_{V-(0)}=(D[[X]])_{D-(0)}$. But

$$
\operatorname{dim}(V[[X]])_{V-(0)}=1,
$$

so if $Q$ is a prime ideal of $D[[X]]$ such that $Q \cap D=(0)$, then $Q$ is minimal in $D[[X]]$. Moreover, it is clear that $Q=Q^{\prime} \cap D[[X]]$ for some minimal prime ideal $Q^{\prime}$ of $V[[X]], Q^{\prime} \neq M[[X]]$. Thus, in order to see that $M[[X]]$ is minimal in $D[[X]]$, it suffices to see that $Q^{\prime} \cap D[[X]] \nsubseteq M[[X]]$ for each such $Q^{\prime}$. Therefore, let

$$
\xi(X)=\sum_{i=0}^{\infty} \xi_{i} X^{i} \in Q^{\prime}-M[[X]]
$$

and let $r$ be the smallest integer for which $\xi_{r} \notin M$. Since $\xi_{r}$ is a unit in $V$, we assume that $\xi_{r}=1$. If $u(X)=\sum_{i=0}^{\infty} \xi_{r+i} X^{i}$, then $u(X)$ is a unit in $V[[X]]$ and

$$
\xi(X) u(X)^{-1}=\left(\sum_{i=0}^{r-1} \xi_{i} X^{i}\right) u(X)^{-1}+X^{r} \in Q^{\prime} \cap D[[X]] .
$$

Hence, $Q^{\prime} \cap D[[X]] \nsubseteq M[[X]]$.

Now let $(0) \subset Q_{1} \subset Q_{2}$ be a chain of prime ideals of $D[[X]]$. Then $Q_{2} \cap D \neq(0)$; hence $Q_{2} \supseteq M[[X]]$. But $M[[X]]$ is minimal, so the containment is proper. It follows that $Q_{2}$ is maximal in $D[[X]$ and that $\operatorname{dim} D[[X]]=2$.

\section{REFERENCES}

1. J. Arnold, On Krull dimension in power series rings, Trans. Amer. Math. Soc., to appear.

2. J. Arnold and J. Brewer, On flat overrings, ideal transforms and generalized transforms of a commutative ring, J. Algebra, 18 (1971), 254-264.

3. D. Fields, Dimension theory in power series rings, Pacific J. Math. 35 (1970), 601611.

4. R. Gilmer, Multiplicative Ideal Theory, Queen's Papers on Pure and Applied Mathematics, Kingston, Ontario, 1968.

5. R. Gilmer and W. Heinzer, Rings of formal power series over a Krull domain, Math. Zeitscher., 106 (1968), 379-387.

6. I. Kaplansky, Commutative Rings, Allyn and Bacon, Boston, 1970.

7. J. Ohm and R. Pendleton, Rings with Noetherian spectrum, Duke Math. J., 35 (1968), 631-640.

8. F. Richman, Generalized quotient rings, Proc. Amer. Math. Soc., 16 (1965), 794799.

9. A. Seidenberg, $A$ note on the dimension thenry of rings. Pacific J. Math., 3 (1953), 505-512. 
10. A. Seidenberg, A note on the dimension theory of rings, II, Pacific J. Math. 4 (1954), 603-614.

Received September 20, 1971.

Virginia Polytechnic Institute

AND

State University 



\section{PACIFIC JOURNAL OF MATHEMATICS}

\section{EDITORS}

H. SAMELSON

Stanford University

Stanford, California 94305

C. R. Hовву

University of Washington

Seattle, Washington 98105
J. DugundJI

Department of Mathematics University of Southern California

Los Angeles, California 90007

RICHARD ARENS

University of California

Los Angeles, California 90024

\section{ASSOCIATE EDITORS}

E. F. BECKENBACH

B. H. NeumanN

F. WOLF

K. YoSHIDA

\section{SUPPORTING INSTITUTIONS}

UNIVERSITY OF BRITISH COLUMBIA

CALIFORNIA INSTITUTE OF TECHNOLOGY

UNIVERSITY OF CALIFORNIA

MONTANA STATE UNIVERSITY

UNIVERSITY OF NEVADA

NEW MEXICO STATE UNIVERSITY

OREGON STATE UNIVERSITY

UNIVERSITY OF OREGON

OSAKA UNIVERSITY
UNIVERSITY OF SOUTHERN CALIFORNIA

STANFORD UNIVERSITY

UNIVERSITY OF TOKYO

UNIVERSITY OF UTAH

WASHINGTON STATE UNIVERSITY

UNIVERSITY OF WASHINGTON

AMERICAN MATHEMATICAL SOCIETY

NAVAL WEAPONS CENTER

Printed in Japan by International Academic Printing Co., Ltd., Tokyo, Japan 


\section{Pacific Journal of Mathematics}

\section{Vol. 44, No. $1 \quad$ May, 1973}

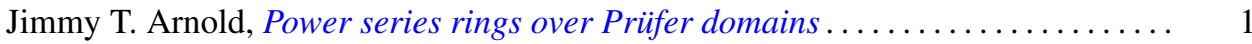

Maynard G. Arsove, On the behavior of Pincherle basis functions . . . . . . . . . 13

Jan William Auer, Fiber integration in smooth bundles ................. 33

George Bachman, Edward Beckenstein and Lawrence Narici, Function algebras

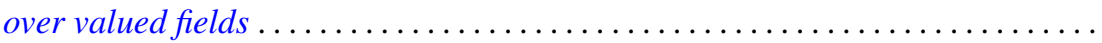

Gerald A. Beer, The index of convexity and the visibility function . . . . . . . . . . .

James Robert Boone, A note on mesocompact and sequentially mesocompact

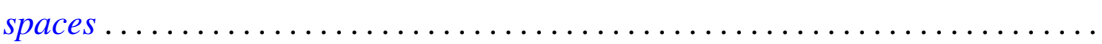

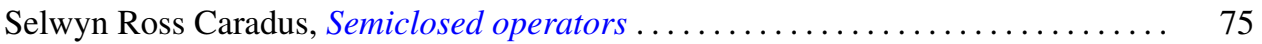

John H. E. Cohn, Two primary factor inequalities . . . . . . . . . . . . . . . 81

Mani Gagrat and Somashekhar Amrith Naimpally, Proximity approach to

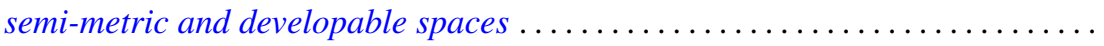

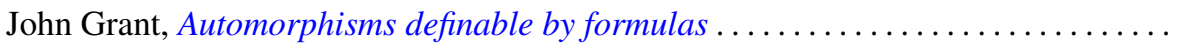

Walter Kurt Hayman, Differential inequalities and local valency ..............

Wolfgang H. Heil, Testing 3-manifolds for projective planes . . . . . . . . . . . . .

107

Melvin Hochster and Louis Jackson Ratliff, Jr., Five theorems on Macaulay

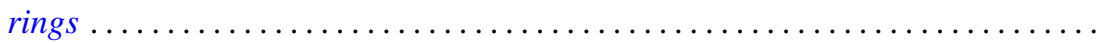

Thomas Benton Hoover, Operator algebras with reducing invariant subspaces ....

James Edgar Keesling, Topological groups whose underlying spaces are separable

Fréchet manifolds...

Frank Leroy Knowles, Idempotents in the boundary of a Lie group . .

191

George Edward Lang, The evaluation map and EHP sequences ...

201

Everette Lee May, Jr, Localizing the spectrum . . . . . . . . . . . .

211

Frank Belsley Miles, Existence of special $K$-sets in certain locally compact abelian groups.

Susan Montgomery, A generalization of a theorem of Jacobson. II . .

T. S. Motzkin and J. L. Walsh, Equilibrium of inverse-distance forces in

three-dimensions.

Arunava Mukherjea and Nicolas A. Tserpes, Invariant measures and the converse

of Haar's theorem on semitopological semigroups .

James Waring Noonan, On close-to-convex functions of order $\beta$

Donald Steven Passman, The Jacobian of a growth transformation

Dean Blackburn Priest, A mean Stieltjes type integral ........ .

Joe Bill Rhodes, Decomposition of semilattices with applications to topological

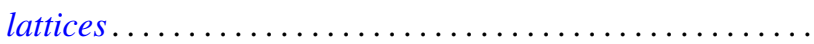

Claus M. Ringel, Socle conditions for $\mathrm{QF}-1$ rings ..........

Richard Rochberg, Linear maps of the disk algebra

Roy W. Ryden, Groups of arithmetic functions under Dirichlet convolution . .

Michael J. Sharpe, A class of operators on excessive functions

Erling Stormer, Automorphisms and equivalence in von Neumann algebras ..

Philip C. Tonne, Matrix representations for linear transformations on series

analytic in the unit disc. 